\title{
A cautionary note on thermal runaway reactions in mixtures of 1-alkyl-3-methylimidazolium ionic liquids and $N$-methylmorpholine- $N$-oxide
}

\author{
Stefan Böhmdorfer (iD - Takashi Hosoya • Thomas Röder • \\ Antje Potthast $\mathbb{D} \cdot$ Thomas Rosenau $(\mathbb{D}$
}

Received: 23 November 2016/ Accepted: 13 March 2017/Published online: 22 March 2017

(C) The Author(s) 2017. This article is an open access publication

\begin{abstract}
N$-Methylmorpholine- $N$-oxide (NMMO) cannot be completely separated by extraction from mixtures with common 1,3-dialkylimidazolium ionic liquids (ILs) due to strong ionic interactions between the two components. At elevated temperatures, above approx. $90{ }^{\circ} \mathrm{C}$, especially under dry conditions and in the presence of acid, alkylating or acylating agents, remaining NMMO in ILs tends to undergo autocatalytic degradation. This is a highly exothermic, unstoppable process that results in explosions, flames, and complete charring of the reaction mixtures. Thus, caution must be exercised when drying or heating ILs that were in
\end{abstract}

\footnotetext{
S. Böhmdorfer - T. Hosoya · A. Potthast ·

T. Rosenau ( $\square)$

Department of Chemistry, University of Natural

Resources and Life Sciences Vienna (BOKU), Muthgasse

18, 1190 Vienna, Austria

e-mail: thomas.rosenau@boku.ac.at

Present Address:

T. Hosoya

Graduate School of Life and Environmental Sciences,

Kyoto Prefectural University, Shimogamo-hangi-cho

11-5, Sakyo-ku, Kyoto-shi, Kyoto, Japan

T. Röder

A13 Lenzing AG, Werkstr. 2, 4860 Lenzing, Austria

A14 e-mail: t.roeder@lenzing.com

T. Rosenau

Johan Gadolin Process Chemistry Centre, Åbo Akademi University, Porthansgatan 3, 20500 Åbo/Turku, Finland
}

previous contact with NMMO, and the absence of amine oxide must be confirmed to avoid potential danger.

Keywords Cellulose $\cdot$ Ionic liquids · NMMO $\cdot$ Binary mixtures $\cdot$ Extractability · Decomposition · Degradation · Exothermicity · Work safety

$\mathrm{N}$-Methylmorpholine- $\mathrm{N}$-oxide (NMMO), as the monohydrate, is well-known as a cellulose solvent and is used industrially in the production of Lyocell. 1-Alkyl3-methylimidazolium ionic liquids (ILs)—with 1-butyl-3-methylimidazolium and 1-ethyl-3methylimidazolium being the most prominent representatives - are much newer, and can be seen as a first generation of ionic liquid-based cellulose solvents. NMMO, apart from its beneficial properties regarding interaction with cellulose, is a strong oxidant and tends to undergo side reactions (Rosenau et al. 2001; Bernier et al. 2009). These might manifest themselves by degradation of the solvent, oxidation of dissolved cellulose (Potthast et al. 2006), discoloration during processing, and yellowing of spun fibers (Rosenau et al. 2005a). There are efficient stabilizers for lyocell dopes available that reliably counteract those effects (Wendler et al. 2008; Rosenau et al. 2005b). Most severe and dangerous, however, is the tendency of NMMO to undergo autocatalytic degradation, with carbenium-iminium ions as the central, catalytically active intermediate in this cycle (Rosenau et al. 1999). 
Once this reaction has started there is no way to stop it again, because of its high exothermicity. Eventually, it will result in complete charring of the mixture, possibly accompanied by explosive degradation, flames, and strong smoke development. Literature accounts describing these effects often use the terms "thermal runaway reaction" or "exothermicity", which are euphemisms for explosive events that in the best case damage lab equipment or production sites or-much worse-might harm health and wellbeing of workers. The degradation mechanisms of NMMO have been comprehensively reviewed (Rosenau et al. 2001); some of them have even been turned into procedures useful in organic synthesis (Rosenau et al. 2004).

1-Butyl-3-methylimidazolium (BMIm) and 1-ethyl-3-methylimidazolium (EMIm), mostly as acetate and chloride, were among the first ionic liquids to be described as cellulose solvents (Swatloski et al. 2002). Most 1,3-dialkylimidazolium ILs are of the 1-alkyl-3-methyl type, so in the following the general term XMIm will be used to described these ILs. Initial optimistic claims of seemingly unlimited applications of these solvents in lignocellulose research have given way to a more realistic view of their advantages and drawbacks. In particular, problems with recycling and byproduct accumulation have cast doubts on their general usefulness in biomass fractionation or pulping, as have literature accounts showing that these solvents undergo reactions with cellulose (Köhler et al. 2007; Liebert et al. 2009; Ebner et al. 2008; Zweckmair et al. 2015) and thus lack the inertness that is often claimed as one of benefits of ILs. Also thermal stress causes slow byproduct formation in XMim ionic liquids, mainly imidazole derivatives (Wendler et al. 2012; Liebner et al. 2010; Dorn et al. 2008).

NMMO has been used in combination with amines or polar organic solvents to achieve cellulose dissolution. Also, ILs have been used as the second component in binary NMMO solvent mixtures, and in turn NMMO has been added to ILs in attempts to further boost their dissolution power (Ingildeev et al. 2013). Most frequently, ILs and NMMO were combined in biorefinery approaches, attempting to pretreat or dissolve biomass to make it amenable to facile hydrolysis to monomeric carbohydrates for later fermentation into platform chemicals or biofuels (Acton 2013; Poornejad et al. 2013; Li 2012; Wikandari et al. (2016); Purwandari et al. 2013; Kuo and Lee 2009).
In this note we would like to direct the readers' attention to the facts that 1-alkyl-3-methylimidazolium ILs (including BMIm and EMIm) retain NMMO which is rendered non-extractable by common organic solvents due to formation of ion pairs, and that the retained NMMO can readily undergo the above-mentioned degradation processes when heated to temperatures above $120{ }^{\circ} \mathrm{C}-\mathrm{e}$.g. in purification attempts-especially at low water contents. CAUTION! Such NMMO residues in the ILs may viciously and rapidly decompose, with all the above described dangerous side effects.

XMIm acetate or chloride, as other ionic liquids, are salts that are liquid at room temperature. They consist of isolated cations and anions interacting by Coulomb forces. NMMO is a zwitterion, also containing an isolated positive and negative charge, but in one molecule. It is an often overlooked fact that ILs and NMMO, both ionic, are predestined to interact by strong Coulomb forces. In fact, the phase diagrams of the binary systems of XMIm/NMMO, and even more the ternary system containing water as third component, are rather complex showing the occurrence of several stable complexes of defined stoichiometry with integer ratios: $x \mathrm{XMIm}$ OAc $\times y$ NMMO with $\{x, y \in \mathbb{N}\}$. In general, the 1,3-dialkylimidazolinium cation is interacting with the negative charge in NMMO, and the resulting cationic complex binds the acetate (chloride) from the IL as counter anion. An in-depth coverage of this topic would by far exceed the scope of this note, and the reader is referred to an upcoming account dealing with that topic in particular. Conformation analysis of NMMO by evaluating NMR coupling constants might be able to shed more light on its molecular interactions in solvent system (Rosenau et al. 2003).

With regard to degradation hazards, the most important consequence of the ionic interaction between IL and NMMO is that the amine oxide cannot be extracted completely from mixtures with XMIm ILs by organic solvents. Table 1 shows the threshold concentrations of NMMO in the respective IL that can be reached by extraction with common organic solvents, starting from 1:1 (wt) mixtures of IL and NMMO. Even repeated extractions or large extractant amounts will not further decrease the NMMO content in the IL below that threshold. Evidently, a higher polarity of the extractant enables it to remove slightly more NMMO from the mixture 
Table 1 Binary mixtures of NMMO with dry 1-alkyl-3-methylimidazolium ionic liquids: dependence of non-extractable NMMO on extractant and IL anion

\begin{tabular}{lllll}
\hline Extractant & EMIM acetate & EMIM chloride & BMIM acetate & BMIM chloride \\
\hline Acetone & 3.5 & 3.8 & 3.6 & 3.8 \\
Chloroform & 3.5 & 3.6 & 3.8 & 4.1 \\
Dichloromethane & 3.8 & 3.8 & 3.8 & 4.0 \\
Ethyl acetate & 4.8 & 5.0 & 4.3 & 4.8 \\
Dioxane & 6.1 & 5.4 & 6.0 & 5.3 \\
Tetrahydrofuran & 6.0 & 5.8 & 6.3 & 5.8 \\
Diethyl ether & 6.3 & 6.1 & 6.5 & 6.1 \\
\hline
\end{tabular}

Given are threshold concentrations of NMMO in the respective IL (wt \%) below which no further extraction of NMMO occurs

with the IL, but the improvements are generally rather small. Chloride ILs retained NMMO slightly better than acetate ILs. Generally, NMMO in binary mixtures with dry XMIm ILs cannot be extracted below contents of $3.5-6 \%$ (w/w), depending on the solvent used for extraction.

Much larger than the effects of the organic extraction medium is the effect of the presence of water. Through its presence, the threshold concentration of non-extractable NMMO is increased: higher water concentrations increase the amount of NMMO retained upon extraction. As a rule of thumb, non-extractable NMMO increases to a molar ratio $\mathrm{H}_{2} \mathrm{O} / \mathrm{IL}$ of approx. 2 and stays constant afterwards, largely independent of the IL's cation (BMIM or EMIM) and anion (chloride or acetate). The nonextractable NMMO in EMIM acetate/NMMO and BMIM chloride/NMMO mixtures in dependence on the water content is shown in Fig. 1 with trichloromethane (chloroform) being two examples of commonly used extractants.

NMMO is well-known to undergo autocatalytic degradation via $N$-methylmorpholinium ions (carbenium-iminium ions) as catalyst. Cleavage of the energy-rich $\mathrm{N}-\mathrm{O}$ bond with concomitant release of a proton from the $N$-methyl group initially forms this intermediate. It then attacks another molecule of NMMO, releasing another catalyst molecule so that the degradation cycle continues (Rosenau et al. 1999). The starting step, cleavage of the $\mathrm{N}-\mathrm{O}$ bond can be triggered by many reagents, both homolytically (radical reactions) and heterolytically (ionic reactions) (Rosenau et al. 2001). The most important inducers are transition metal ions, as well as alkylating and acylating agents that attack the negatively

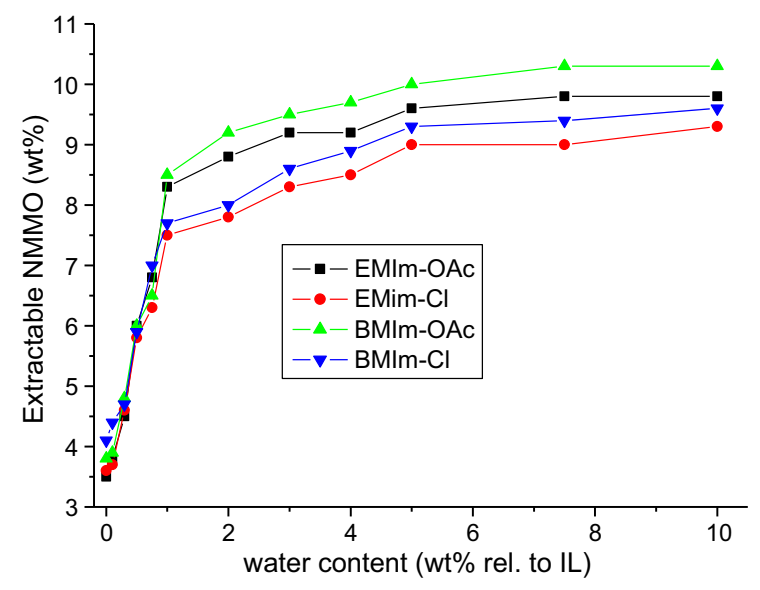

Fig. 1 Non-extractable NMMO in IL/NMMO mixtures in dependence on the water content; extractant: trichloromethane

charged oxygen in NMMO (so-called Polonowskitype reactions) (Rosenau et al. 2002). The residual, non-extractable NMMO in ionic liquids can undergo such reactions as well as pure NMMO does, and grave danger is posed when an IL is erroneously considered to be NMMO-free, and the experimentalist is not aware of the residual NMMO content.

The instability of NMMO in mixtures can be readily tested by the onset temperature, i.e. the temperature at which the autocatalytic degradation starts and becomes uncontrollable. The reaction is usually carried out with volumes of $1-3 \mathrm{~cm}^{3}$ in explosion-proof autoclaves or calorimeters that are heated up at controlled rates. It is surprisingly accurate and repeatable with errors of less than $5^{\circ}$ $\mathrm{C}$, provided that the heating rates are similar. Table 2 lists the onset temperatures of different IL/NMMO mixtures. CAUTION! NMMO in 1-alkyl-3- 
Table 2 Onset temperatures $\left({ }^{\circ} \mathrm{C}\right)$ for the uncontrolled autocatalytic degradation of NMMO in NMMO/IL mixtures of different composition

\begin{tabular}{|c|c|c|c|c|c|}
\hline IL/NMMO (wt\%) & Additive (wt\% rel. to IL) & EMIM chloride & EMIM acetate & BMIM chloride & BMIM acetate \\
\hline $95 / 5$ & None (freshly purified IL, see text) & 228 & 235 & 214 & 238 \\
\hline $95 / 5$ & IL heated to $200{ }^{\circ} \mathrm{C}$ for $2 \mathrm{~h}$ before & 172 & 144 & 179 & 150 \\
\hline $95 / 5$ & IL heated to $200{ }^{\circ} \mathrm{C}$ for $24 \mathrm{~h}$ before & 108 & 92 & 114 & 96 \\
\hline $95 / 5$ & 0.1 , acetic acid & 154 & 162 & 164 & 149 \\
\hline $95 / 5$ & 0.1 , gluconic acid & 149 & 149 & 154 & 152 \\
\hline $95 / 5$ & $0.1, N$-methylmorpholine & 237 & 240 & 242 & 239 \\
\hline $95 / 5$ & 0.1, 1-methylimidazole & 228 & 244 & 248 & 239 \\
\hline $95 / 5$ & 0.1 , imidazole & 224 & 111 & 230 & 108 \\
\hline $95 / 5$ & 0.2 , imidazole & 228 & 114 & 226 & 110 \\
\hline $95 / 5$ & $0.01,1$-acetylimidazole & 94 & 90 & 98 & 96 \\
\hline $95 / 5$ & 0.01 , acetic anhydride & 103 & 99 & 97 & 105 \\
\hline $95 / 5$ & 0.01 , butyl iodide & 127 & 164 & 130 & 167 \\
\hline
\end{tabular}

Lower onset temperatures mean more unstable mixtures. Additives were admixed to simulate the effects of degradation products and $\mathrm{pH}$. Values give the average of three independent runs, with deviations between the runs being generally $<8{ }^{\circ} \mathrm{C}$

methylimidazolium ionic liquids cannot be considered safe at temperatures above $90{ }^{\circ} \mathrm{C}$.

Highest stabilities are obtained in pure ILs from which degradation productions had previously been removed (Liebner et al. 2010). Acidic degradation products, such as acetic acid, gluconic acid from cellulose or 2-furancarboxylic acid from xylan, generally destabilize NMMO, whereas basic degradation products, such as 1-methylimidazole from the IL (Liebner et al. 2010) or $N$-methylmorpholine from NMMO, render it more stable, in complete agreement with general NMMO chemistry. There is one important exception: imidazole has a pronounced destabilizing effect in XMIm acetates (BMIM-OAc, EMIM-OAc). It reacts with the acetate to form small equilibrium amounts of 1-acetylimidazole, which, in turn, is a strong acetyl transfer agent that acylates the amine $\mathrm{N}$-oxide oxygen and induces immediate NMMO decomposition (Zweckmair et al. 2015). This effect is evidently only seen in acetate ILs (and in other ILs with carboxylic acid anions that can form 1-acylimidazoles), but not in chloride ILs.

Interestingly, while the presence of water usually stabilized NMMO, stabilization is much less for IL/ NMMO mixtures (see Fig. 2). Addition of water increases the decomposition onset temperature only slightly, and only relatively high water contents cause the degradation to start at significantly higher temperatures-above a molar ratio $\mathrm{H}_{2} \mathrm{O} / \mathrm{IL}$ of approx. 2,

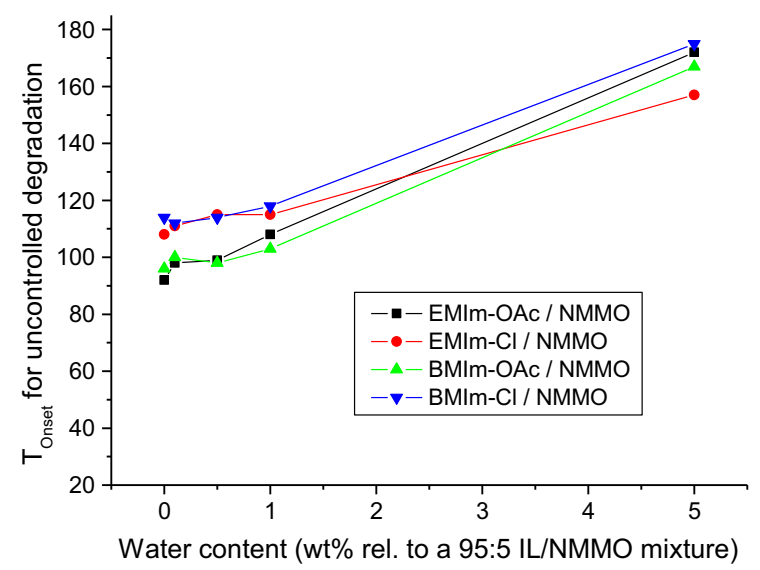

Fig. 2 Influence of the water content in IL/NMMO mixtures on the onset temperature $\left(\mathrm{T}_{\text {onset }}\right)$ of the uncontrolled thermal degradation

by analogy to the above extraction experiments. This means, on the other hand, that drying (water capture) of an IL/NMMO mixture will decrease its overall stability, which is potentially dangerous if the temperature of the mixture is above $90{ }^{\circ} \mathrm{C}$ and thus in the range of possible NMMO degradation.

A good demonstration experiment uses a mixture of EMIM-OAc/NMMO/water 90/5/5 (w/w), the IL having been "aged" by thermal treatment at $200{ }^{\circ} \mathrm{C}$ over $24 \mathrm{~h}$ (see Table 2). At $120^{\circ} \mathrm{C}$, this ternary system is stable over several hours; no water is 
removed as it is tightly bound by NMMO and IL as "hydrates". If anhydrous magnesium sulfate-a seemingly "innocent" drying agent—is added (equimolar to the water amount), the mixture will get dark within about 2 min and violently decompose a few seconds later: the protective effect of large water amounts is undone by the $\mathrm{MgSO}_{4}$ drying agent, and the mixture is in the temperature zone above $90{ }^{\circ} \mathrm{C}$ that is safe at higher water contents, but unsafe when no water as third component is present.

Special care must be taken to avoid the simultaneous presence of NMMO and acylating agents, e.g. if an acetylation is to be carried out in an IL, while the IL unknowingly contains NMMO as carry-over from previous experiments.

Alkylating agents must also be viewed critically; there have been incidents with NMMO decomposition when NMMO was admixed to freshly prepared (and thus allegedly pure) BMIM IL. Since the IL in fact had still contained traces of butyl halide from its manufacture (quaternization of 1-methylimidazole into BMIM), a pronounced instability of NMMO was triggered. The safest way to prevent such accidents is to ensure the absence of NMMO when heating an IL. Contamination with NMMO cannot be detected by GC (no volatility of both IL and NMMO) or NMR (concentration too low), so that HPLC and CE (capillary electrophoresis) (Potthast et al. 2000) seem to be the methods of choice. NMMO traces, if present, can be easily reduced by aqueous ferrous sulfate or sodium borohydride, giving innocent and readily removable $\mathrm{N}$-methylmorpholine.

We hope that these cautionary remarks will help to increase awareness of the inherent dangers of working with NMMO at elevated temperatures, and to thus prevent any related accidents in laboratories.

Acknowledgments Open access funding provided by University of Natural Resources and Life Sciences Vienna (BOKU). The authors thank the Japanese Society for Promotion of Science (JSPS) for support of the work through travel grants.

Open Access This article is distributed under the terms of the Creative Commons Attribution 4.0 International License (http://creativecommons.org/licenses/by/4.0/), which permits unrestricted use, distribution, and reproduction in any medium, provided you give appropriate credit to the original author(s) and the source, provide a link to the Creative Commons license, and indicate if changes were made.

\section{References}

Acton QA (ed) (2013) Advances in ethanol research and application. Scholarly editions(TM), Atlanta, Georgia, p 538

Bernier D, Wefelscheid UK, Woodward S (2009) Properties, preparation and synthetic uses of amine N-oxides. An update. Org Prep Proc Int 41(3):175-210

Dorn S, Wendler F, Meister F, Heinze T (2008) Interactions of ionic liquids with polysaccharides -7 : thermal stability of cellulose in ionic liquids and $N$-methylmorpholine- $N$-oxide. Macromol Mater Eng 293(11):907-913

Ebner G, Schiehser S, Potthast A, Rosenau T (2008) Side reaction of cellulose with common 1-alkyl-3-methylimidazoliumbased ionic liquids. Tetrahedron Lett 49:7322-7324

Ingildeev D, Effenberger F, Bredereck K, Hermanutz F (2013) Comparison of direct solvents for regenerated cellulosic fibers via the Lyocell process and by means of ionic liquids. J Appl Polym Sci 128(6):4141-4150

Köhler S, Liebert T, Schöbitz M, Schaller J, Meister F, Günther W, Heinze T (2007) Interactions of ionic liquids with polysaccharides. 1. Unexpected acetylation of cellulose with 1-ethyl-3-methylimidazolium acetate. Macromol Rapid Commun 28:2311-2317

Kuo CH, Lee CK (2009) Enhanced enzymatic hydrolysis of sugarcane bagasse by $N$-methylmorpholine- $N$-oxide pretreatment. Biores Technol 100(2):866-871

Li EY (2012) Enzymatic hydrolysis of cellulose pretreated with ionic liquids and $N$-methylmorpholine- $N$-oxide, MSc. Thesis. Florida State University

Liebert T, Heinze T, Edgar K (eds) (2009) Cellulose solvents; for analysis, shaping and chemical modification. ACS symposium series, vol 1033. American Chemical Society, Washington, DC. doi:10.1021/bk-2010-1033.ch008

Liebner F, Patel I, Ebner G, Becker E, Horix M, Potthast A, Rosenau T (2010) Thermal aging of 1-alkyl-3-methylimidazolium ionic liquids and its effect on dissolved cellulose. Holzforschung 64(2):161-166

Poornejad N, Karimi K, Behzad T (2013) Improvement of saccharification and ethanol production from rice straw by NMMO and [BMIM][OAc] pretreatments. Ind Crops Prod 41:408-413

Potthast A, Rosenau T, Kosma P, Schelosky N, Baldinger T (2000) Thermal reactions of $N$-methylmorpholine- $N$-oxide (NMMO): a general method for separation and quantification of $N$-methylmorpholine- $N$-oxide and its main degradation products $N$-methylmorpholine and morpholine by capillary electrophoresis (CE). Holzforschung 54(6):641-646

Potthast A, Rosenau T, Kosma P (2006) Analysis of oxidized functionalities in cellulose. Adv Polym Sci 205:1-48

Purwandari FA, Sanjaya AP, Millati R, Cahyanto MN, Horváth IS, Niklasson C, Taherzadeh MJ (2013) Pretreatment of oil palm empty fruit bunch (OPEFB) by $N$-methylmorpholine- $N$-oxide (NMMO) for biogas production: structural changes and digestion improvement. Biores Technol 128:461-466

Rosenau T, Potthast A, Kosma P, Chen CL, Gratzl JS (1999) Autocatalytic decomposition of $\mathrm{N}$-methylmorpholine- $\mathrm{N}$ - 
oxide induced by Mannich intermediates. J Org Chem 64:2166-2167

Rosenau T, Potthast A, Sixta H, Kosma P (2001) The chemistry of side reactions and byproduct formation in the system NMMO/cellulose (Lyocell process). Progr Polym Sci 26(9):1763-1837

Rosenau T, Potthast A, Hofinger A, Sixta H, Kosma P (2002) Instabilities in the system NMMO/water/cellulose (Lyocell process) caused by Polonowski type reactions. Holzforschung 56(2):199-208

Rosenau T, Hofinger A, Potthast A, Kosma P (2003) On the conformation of the cellulose solvent $N$-methylmorpholine- $N$-oxide (NMMO) in solution. Polymer 44(20):6153

Rosenau T, Hofinger A, Potthast A, Kosma P (2004) A general, selective high-yield $\mathrm{N}$-demethylation procedure for tertiary amines by solid reagents in a convenient column chromatography-like setup. Org Lett 6(4):541-544

Rosenau T, Potthast A, Milacher W, Adorjan I, Hofinger A, Kosma P (2005a) Discoloration of cellulose solutions in $N$-methyl morpholine- $N$-oxide (Lyocell). Part 2: isolation and identification of chromophores. Cellulose 12(2):197208

Rosenau T, Schmid P, Potthast A, Kosma P (2005b) Stabilization of cellulose solutions in $N$-methylmorpholine- $N$ - oxide (Lyocell dopes) by addition of an $N$-oxide as sacrificial substrate. Holzforschung 59(5):503-506

Swatloski RP, Spear SK, John D, Holbrey JD, Rogers RD (2002) Dissolution of cellulose with ionic liquids. J Am Chem Soc 124:4974-4975

Wendler F, Konkin A, Heinze T (2008) Studies on the stabilization of modified Lyocell solutions. Macromol Symp 262(1):72-84

Wendler F, Todi L-N, Meister F (2012) Thermostability of imidazolium ionic liquids as direct solvents for cellulose. Thermochim Acta 528:76-84

Wikandari R, Millati R, Taherzadeh MJ (2016) Pretreatment of lignocelluloses with solvent $\mathrm{N}$-methylmorpholine $\mathrm{N}$-oxide. In: Musatto SI (ed) Biomass fractionation technologies for a lignocellulosic feedstock based biorefinery, 1st edn. Elsevier, Amsterdam, pp 255-280

Zweckmair T, Hettegger H, Abushammala H, Bacher M, Potthast A, Laborie M-P, Rosenau T (2015) On the mechanism of the unwanted acetylation of polysaccharides by 1,3-dialkylimidazolium acetate ionic liquids. Part 1: analysis, acetylating agent, influence of water, and mechanistic considerations. Cellulose 22(6):3583-3596 